\title{
DUST DRIVEN YINDS IN LATE SUPERGIANTS
}

Erwin Sedlmayr

Carsten Dominik

Institut für Astronomie und Astrophysik

Technische Universität Berlin

Hardenbergstraße 36, D-1000 Berlin 12
Hans-Peter Gail

Institut für Theoretische Astrophysik Universität Heidelberg

Im Neuenheimer Feld 561, 6900 Heidelberg 1

\section{Introduction}

The cool extended shells of giants and supergiants are well known to be places of copious dust formation as indicated by the occurrence of pronounced extinction, reddening, and polarization of the continuous star light and by the appearance of particular absorption features, both manifesting the interaction of photons with particles considerably larger than atoms or molecules.

The accepted explanation of these phenomena is the formation of circumstellar dust, i.e. small solid particles with a typical size of the order of 0.1 micron. However, the analysis of these effects yields only information on the interaction of photons with certain functional groups within the clusters - e.g. Si-O, C-H, C-C bending and $\mathrm{Si}-\mathrm{O}, \mathrm{C}-\mathrm{C}, \ldots$ stretching vibrations - and thus allows no definite determination of the "real" physical structure and chemical composition of the grain particles. Therefore, observational conclusions concerning the properties of circumstellar dust can provide only some "mean" information which allows no definitive conclusions regarding the true nature of the observed grains (geometrical shape, crystalline structure, ...?).

An adequate answer to these questions must be based on the detailed study of the processes of formation and growth of dust particles in these environments. However, dust formation cannot be considered as an isolated problem because due to their huge absorption cross sections even a small contamination of the atmospheres by circumstellar dust may have a significant influence on the radiative transfer and (via energy - and momentum-coupling) on the thermodynamic and hydrodynamic structure of the dust forming shell.

Therefore, a consistent modelling of such objects requires the treatment of the coupled nonlinear system comprising

- radiative transfer

- density, velocity, temperature structure of the shell

- chemistry and dust formation.

This has been pointed out by several authors in the past (e.g. Wlokramasinghe, 1972; Kwok, 1975; Salpeter, 1974; Deguchi, 1980; Jura, 1983), but 
there was no consistent treatment of all relevant aspects of this important problem (especially for the complex of dust formation and growth) until the papers of Kozasa et al. (1984) and Gail and Sedlmayr (1984), who addressed the problem of dust driven winds for M-type stars and C-type stars, respectively.

Before presenting the self consistent theoretical formulation of the problem, let us have a look on the observational facts providing a general characterization of such objects (e.g. Knapp et al., 1982, 1986; Rowan-Robinson and Harris, 1983):

- typical luminosities $10^{3} \ldots 10^{5} \mathrm{~L}_{0}$

- $\quad$ stellar masses $0.7 \ldots 2 \mathrm{M}_{\odot}$

- " mass loss rates $10^{-7} \ldots 10^{-4} \mathrm{M}_{\odot} \mathrm{y} \mathrm{r}^{-1}$

- " wind velocities $10 \ldots 30 \mathrm{~km} \mathrm{~s}^{-1}$

- " mass of the shells $0.1 \ldots 0.6 \mathrm{M}_{\odot}$

- wind phenomenon lasts for at least $10^{4}$ years, no abrupt ejection of material.

\section{The basic equations}

For giants and supergiants the relevant timescales which describe the evolution of the atmosphere generally are large compared to the characteristic timescales which control dust formation and growth and their thermodvnamic and hydrodynamic feedback to the atmosphere. Therefore, we restrict our discussion to the stationary case. Further we assume a spherically symmetric outflow which beyond a certain distance from the star becomes cool enough for effective dust formation to occur. This assumption of spherical symmetry is not trivial in view of the nucleation process which is extremely sensitive to the temperature. Thus even small temperature fluctuations might have a large influence on the local nucleation rate and on the local atmospheric structure (cloud formation?).

\subsection{The model equations}

The hydrodynamical equations for the flow are given by the equation of mass conservation

$$
\dot{\mathrm{M}}=4 \pi r^{2} \rho \mathrm{v}
$$

and the equation of momentum conservation

$$
v \frac{\partial v}{\partial r}=-\frac{1}{\rho} \frac{\partial p}{\partial r}-\frac{G M_{*}}{r^{2}}(1-\alpha)
$$

with mass density $\rho$, hydrodynamical velocity $v$, thermodynamical pressure $p$ and radiative acceleration $\alpha$ (normated to gravitation). It is assumed that the total mass contained in the wind is negligibly small compared to the stellar mass $M_{*}$. Due to the assumption of stationarity, the mass loss rate $\dot{M}$ is a constant during the 
considered evolutionary state of the star.

By the equation of state

$$
\mathrm{p}=\mathrm{nkT}
$$

the gas pressure $p$ is expressed as function of the total number density $n$ and the gas kinetic temperature $T$. In the considered case

$$
\mathrm{n}=\mathrm{n}_{\mathrm{H}}+\mathrm{n}_{\mathrm{H}}+\mathrm{n}_{\mathrm{He}}
$$

is a reliable approximation.

The temperature structure is determined by adopting radiative equilibrium in the shell. For optical thin winds (this condition is fulfilled for all objects discussed in the present paper) $T(r)$ is given analytically according to Lucy (1976)

$$
T^{4}(r)=\frac{1}{2} T_{*}^{4} \frac{K_{J}}{K_{B}}\left\{1-\left[1-\left(\frac{R_{*}}{r}\right)^{2}\right]^{1 / 2}+\frac{3}{2} \tau_{L}\right\}
$$

with effective temperature $T_{*}$, stellar radius $R_{*}$ and the "diluted" optical depth

$$
\left.\tau_{\mathrm{L}}=-\int_{r}^{\infty} \mathrm{d} r^{\prime} \kappa_{\mathrm{H}}\left(r^{\prime}\right) \frac{\mathrm{R}_{*}}{\left(\frac{r^{\prime}}{2}\right.}\right)^{2} .
$$

$\mathrm{KJ}, \mathrm{KB}$ and $\mathrm{KH}$ are the intensity-, the Planck- and the flux-mean of the monochromatic extinction coefficient $\kappa_{V}$, respectively. With $T_{*}$ and $R_{*}$ also the stellar luminosity

$$
L_{*}=4 \pi R_{* \sigma}^{2} T_{*}^{4}
$$

is given by the Stefan-Boltzmann law.

The external parameters of the problem are $M_{*}, T_{*}, L_{*}$ and $\dot{M}$. However, in a consistent treatment the optical depth $\tau$ L is not only subject to the boundary condition

$$
\tau_{L}(\infty)=0
$$

but also to the additional condition

$$
\tau_{L}\left(R_{*}\right)=\frac{2}{3}\left(2 \frac{K_{B}}{K_{J}}-1\right)
$$

in order to guarantee

$$
T\left(R_{*}\right)=T_{*}
$$

Hence, one of the above parameters is determined by an eigenvalue problem. For this reason, the problem is completely determined by specification of only three independent stellar parameters.

\subsection{The equations for the dust}

For describing dust formation and growth we adopt a frame of reference which is moving with the flow. Then the system of moment equations which describes these processes in a sufficient approximation reduces to 


$$
\frac{d k_{0}}{d r}=\frac{j_{*}^{s}}{v} \quad \text { (cluster formation) }
$$

and

$$
\frac{d k_{i}}{d r}=\frac{i}{v_{\tau}} k_{i-1} \quad i=1,2,3 \quad \text { (grain growth) }
$$

with $j_{*}^{S}=v r^{2} J_{*}^{S}$ and $k_{i}=v r^{2} K_{i}$ where $J_{*}^{S}$ is the stationary nucleation rate and

$$
k_{i}=\int_{1}^{\infty} d N^{i / 3} f(t, r, N)
$$

the $i-t h$ moment of the local particle distribution function $f(t, r, N)$ describing the number density of ciusters consisting of $\mathbb{N}$ monomers (Gail and Sedlmayr, 1987c).

The assumption of a stationary nucleation rate is justified by the fact that in situations not too far from equilibrium the relaxation time is small compared to those timescales which determine the hydrodynamical evolution of the shell. Yet this is not true for the growth process which always has to be treated as a time dependent problem (c.f. Gail and Sedlmayr, 1987c).

The treatment of grain formation and growth is of fundamental importance within the context of our problem. In the last decade, considerable progress has been achieved in this field based on the pioneering work of the Cornell group (Salpeter et al., Draine) and the Kyoto group (Hasegasa, Yamamoto, Kozasa, Seki).

All approaches are based either on the thermodynamical description of the gassolid phase transition by classical nucleation theory or on a detailed discussion of the relevant chemical reactions leading finally to critical clusters (e.g. review by Gail, Sedlmayr, 1987d). We will refrain from a presentation of these various approaches but only list the basic molecules from which the primary condensates are likely to be formed:

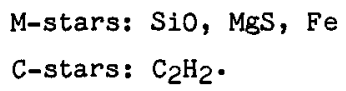

All calculations presented in the following chapters are restricted to dust formation in C-stars and are performed under the assumption of chemical equilibrium for the molecular reactions among the various chemical elements. The element abundances have to be specified as additional external parameters. For dust driven winds the amount of condensed material determines the velocity field and in particular the terminal velocity of the wind. For this reason, the abundances of the dust forming elements and the terminal velocity of the wind are coupled very closely. For the case of M-stars similar calculations have been performed by Kozasa et al. ( 1984 ) considering formation of $\mathrm{MgSiO}_{3}-$ grains. 
In order to demonstrate the typical results of a selfconsistent calculation, the detailed wind model of an arbitrarily selected star with the parameters $M_{*}=1 M_{\Theta}$, $T_{*}=2000 \mathrm{~K}, L_{*}=3 \cdot 3 \cdot 10^{4} \mathrm{~L}_{\odot}$ and a carbon/oxygen abundance ratio $\varepsilon_{C} / \varepsilon_{0}=2$ (and cosmic abundances otherwise) is depicted. The gas extinction coefficients used for the radiative transfer calculations are adopted from Scholz and Tsuji (1984).

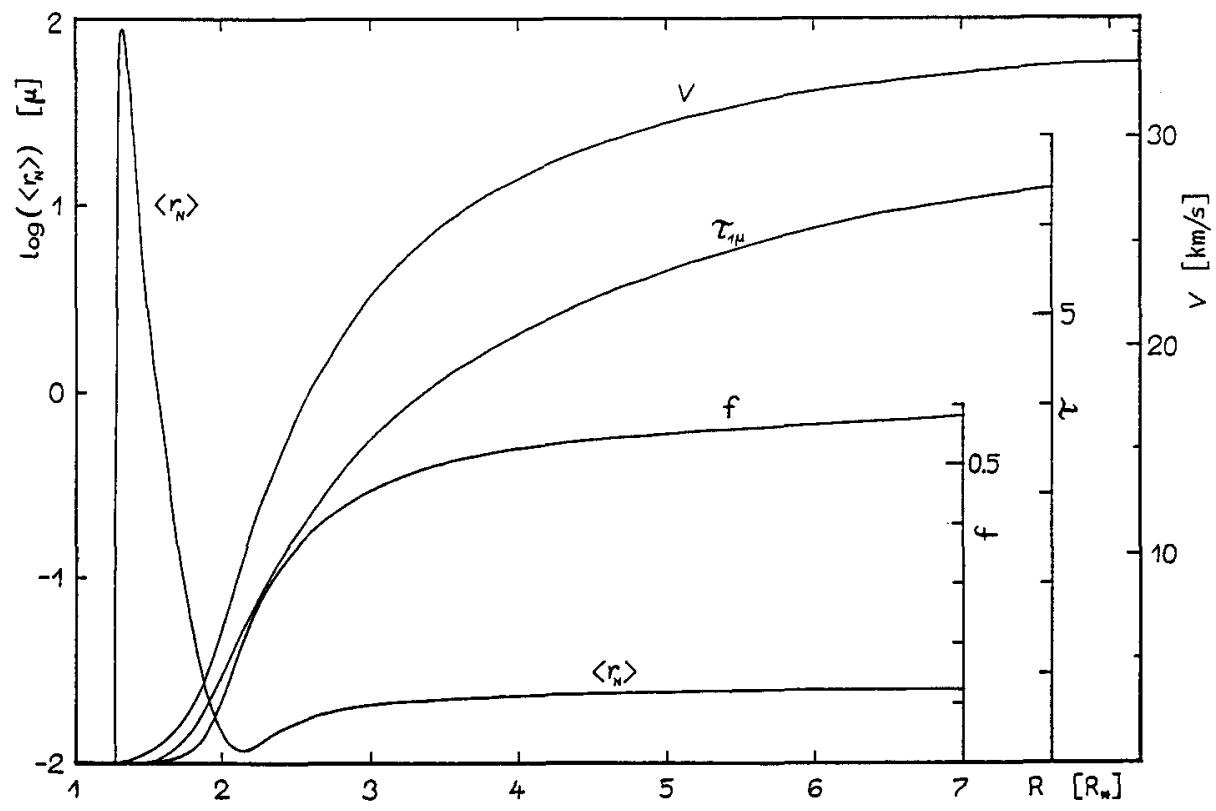

Fig. 1: Structure of a dust driven wind: mean grain radius $\left\langle r_{N}\right\rangle$, hydrodynamical velocity $v$, optical depth $\tau_{1 \mu}$ of the dust shell at $\lambda=1 \mu$, degree of condensation $\mathrm{f}$.

The eigenvalue problem is solved for $\dot{M}$ which results to be $3.5 \cdot 10^{-5} \mathrm{M}_{\odot} \mathrm{yr}^{-1}$. Fig. 1 shows the structure of the dust shell as it has been discussed in former papers (Gail, Sedlmayr, 1985, 1987a). We only want to point out two facts:

- hydrodynamics and dust formation are coupled so strongly that a consistent treatment is necessary,

- the mean grain radius is very small. Only very few big particles are formed in the early phase of the condensation process.

\section{The influence of the model parameters}

Our calculations allow to give an overview over the dust driven wind solutions in the case of C-stars and their dependence on the four parameters of the system of equations. 


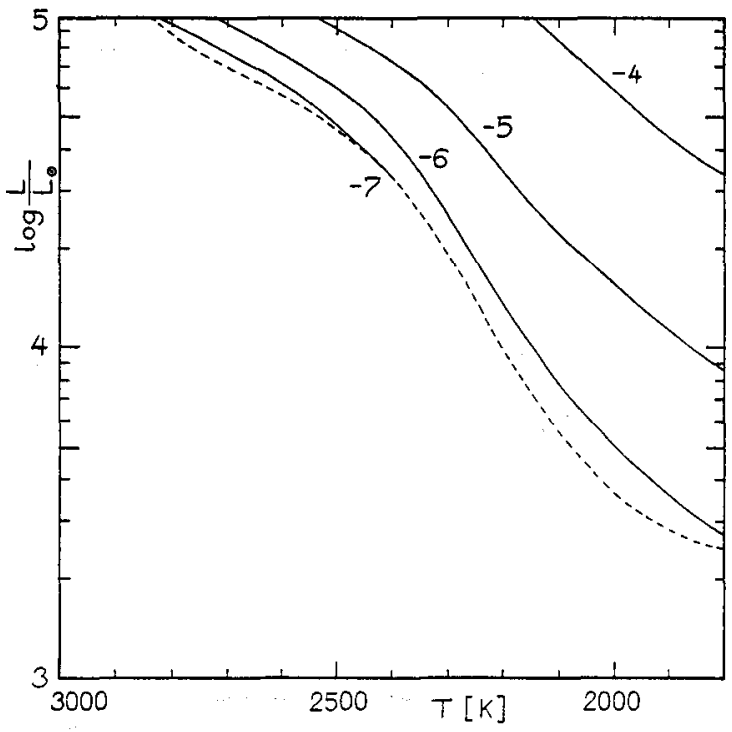

Fig. 2:

HR-diagram with lines of constant mass loss rate. The numbers indicate $\log \mathrm{M}\left(\mathrm{M}_{\mathrm{G}} \mathrm{yr}^{1}\right)$. Broken line: limit line for dust driven winds.

Fig. 2 shows an HR-diagram with contour lines of constant mass loss rates. The lines have been calculated for $M_{*}=1 M_{\ominus}$ and $\varepsilon_{C} / \varepsilon_{0}=2$. The dust driven winds are confined to a well defined area in the upper right corner of the HRD. Since effective dust formation requires sufficient high densities, there exists a minimum mass loss rate of about $10^{-7} \mathrm{M}_{\odot} \mathrm{yr}^{-1}$ (Gail, Sedlmayr, 1987b). Because of the obvious correlation between luminosity and mass loss, this defines a limit line (the broken line in Fig. 2). Below this line no dust driven winds are possible. This fact is in keeping with the observation that C-stars with extended envelopes always have mass loss rates above $10^{-7} \mathrm{M}_{\odot} \mathrm{yr}$ (Knapp and Morris, 1985).

As can be seen from Fig. 2, the mass loss rate increases strongly with increasing luminosity and decreasing temperature.

Similar HR-diagrams can be constructed for other quantities. The terminal velocity ranges from $10 \mathrm{~km} \mathrm{~s}^{-1}$ to about $40 \mathrm{~km} \mathrm{~s}^{-1}$. This again is in good agreement with observations by Knapp and Morris (1985) who yield velocities from $8 \mathrm{~km} \mathrm{~s}^{-1}$ up to 30 $\mathrm{km} \mathrm{s} \mathrm{s}^{-1}$. vo increases with increasing luminosity.

The degree of condensation, i.e. the fraction of condensable material actually condensed into grains, is always greater than $10 \%$ and increases with decreasing temperature. The mean value resulting for the dust to gas ratio by mass in the wind is $2.8 \cdot 10^{-3}$, which is in excellent agreement with the value given by Knapp (1985b).

The mean grain radius results to be rather small. In our calculations it ranges from $0.005 \mu$ up to $0.05 \mu$ which is significantly smaller than the size interval from $0.1 \mu$ up to $10 \mu$ which is usually adopted. The size of the grains increases with decreasing effective temperature of the star. 
Variation of the stellar mass mainly affects the mass loss rate. For fixed $I_{*}$, $L_{*}$, $\varepsilon_{\mathrm{C}} / \varepsilon_{0}$ the mass loss rate increases almost exponentially with decreasing mass. This effect, combined with the temperature and luminosity dependencies, strongly suggests that dust formation might be one possible driving mechanism for the so called superwind postulated for the AGB-PN transition.

Condensation degree, mean grain radius and the terminal velocity show only weak dependencies on the stellar mass.

\subsection{The influence of $\varepsilon_{c} / \varepsilon_{0}$}

Variations of the overabundance of carbon relative to oxygen mainly affects the terminal velocity. For fixed $T_{*}, L_{*}, M_{*}$ these two quantities are almost in linear correlation. This fact allows to determine $\varepsilon_{C} / \varepsilon_{0}$ by observing $v_{\infty}$. To fit the observed velocities (vo< $30 \mathrm{~km} \mathrm{~s}^{-1}$ ), $\varepsilon_{\mathrm{C}} / \varepsilon_{0}$ has to be smaller than about 2 .

An increase of $\varepsilon_{C} / \varepsilon_{0}$ also leads to a decreasing mean radius of the dust grains caused by the greater production rate of small clusters and the short growth time. The mass loss rate and the condensation degree show only very week dependencies on $\varepsilon_{\mathrm{C}} / \varepsilon_{0}$

The results of this chapter will be discussed in detail in a forthcoming paper.

\section{References}

Deguchi, S.: 1980, Astrophys. J. 236,567

Gail, H.-P., Keller, R., Sedlmayr, E.: 1984, Astron. Astrophys. 133, 320

Gail, H.-P., Sedlmayr, E.: 1987a, Astron. Astrophys. 171, 197

Gail, H.-P., Sedlmayr, E.: 1987b, Astron. Astrophys. 177, 186

Gail, H.-P., Sedlmayr, E.: 1987c, in preparation

Gail, H.-P., Sedlmayr, E.: 1987d, In "Physical Processes in Interstellar Clouds", eds. G.E. Morfil, M. Scholer, NATO ASI Series

Jura, M.: 1983, Astrophys. J. 267, 647

Knapp, G.R., Phillips, T.G., Leighton, R.B., Lo, K.Y., Wannier, P.G.: 1982, Astrophys. J. 252,616

Knapp, G.R., Morris, M.: 1985a, Astrophys. J. 292, 640

Knapp, G.R.: 1985b, Astrophys. J. 293, 273

Knapp, G.R.: 1986, Astrophys. J. $3 \frac{11}{11}, 731$

Kozasa, T., Hasegawa, H., Seki, J.: 1984, Astrophys. Space Sci. 98, 61

Kwok, S.: 1975, Astrophys. J. 198583

Lucy, L.B.: 1976, Astrophys. J. 198, 583

Rowan-Robinson, M., Harris, S.: 1983 , Mon. Not. R. astron. Soc. 202, 797

Salpeter, E.E.: 1974a, Astrophys. J. 193, 579

Scholz, M., Tsuji, T.: 1984, Astron. Astrophys. 130, 11

Wickramasinghe, N.C.: 1972, in "Interstellar Matter", eds. N.C. Wickramasinghe, F.D. Kahn, P.G. Mezger, Geneva Observ., Sauverny 